\title{
Resolution of plaque-type psoriasis: what is left behind (and reinitiates the disease)
}

\author{
Theresa Benezeder ${ }^{1,2} \cdot$ Peter Wolf ${ }^{1}[$
}

Received: 16 July 2019 / Accepted: 27 September 2019/Published online: 31 October 2019

(C) The Author(s) 2019

\begin{abstract}
Psoriasis is a chronic inflammatory skin disease that involves numerous types of immune cells and cytokines resulting in an inflammatory feedback loop and hyperproliferation of the epidermis. A more detailed understanding of the underlying pathophysiology has revolutionized anti-psoriatic treatment and led to the development of various new drugs targeting key inflammatory cytokines such as IL-17A and IL-23. Successfully treated psoriatic lesions often resolve completely, leaving nothing visible to the naked eye. However, such lesions tend to recur within months at the exact same body sites. What is left behind at the cellular and molecular levels that potentially reinitiates psoriasis? Here, we elucidate the cellular and molecular "scar" and its imprints left after clinical resolution of psoriasis treated with anti-TNF $\alpha$, anti-IL-17, or anti-IL-23 antibodies or phototherapy. Hidden cytokine stores and remaining tissue-resident memory T cells (TRMs) might hold the clue for disease recurrence.
\end{abstract}

Keywords IL-17 · IL-23 $\cdot$ Phototherapy $\cdot$ Tissue-resident memory T cells (TRMs) $\cdot$ Molecular scar $\cdot$ Recurrence $\cdot$ Psoriasis

\section{Background}

Psoriasis is a chronic skin disease that results from a multifaceted interaction of immunological, environmental, and genetic factors $[1,2]$. According to a recent systematic review, the global prevalence of psoriasis ranges from 0.09 to $5.1 \%$ [3]. The most common form of psoriasis, occurring in $85-90 \%$ of all patients, is plaque-type psoriasis characterized by well-demarcated, scaly and erythematous, infiltrated plaques $[1,4]$. On the microscopic level, keratinocytes proliferate rapidly at high turnover rates, which leads to incomplete terminal differentiation. Abnormal differentiation causes thickening of the epidermis (acanthosis), retention of keratinocyte nuclei in the stratum corneum (parakeratosis), and loss of the granular layer. Neutrophils accumulate in the epidermis to form Munro's microabscesses. Psoriatic lesions are highly vascular

This article is a contribution to the special issue on Resolution of Inflammation in Chronic Diseases - Guest Editor: Markus Neurath

Peter Wolf

peter.wolf@medunigraz.at

1 Department of Dermatology, Medical University of Graz, Auenbruggerplatz 8, 8036 Graz, Austria

2 Center for Medical Research, Medical University of Graz, Graz, Austria and also densely infiltrated by T cells and dendritic cells (DC) $[2,5,6]$ which are key players in its pathophysiology.

\section{Pathophysiology of psoriasis}

Various external factors like trauma and injury, infection, or medication can stress or damage keratinocytes [4]. Stressed or dying cells release nucleic acids along with the antimicrobial peptide (AMP) LL-37. LL-37 causes the loss of tolerance to self-nucleic acids and forms complexes with self-DNA/-RNA. These complexes can activate plasmacytoid dendritic cells (pDCs) via toll-like receptor 7 (TLR7) [7]. pDCs mainly produce type 1 interferons (IFN- $\alpha$, IFN- $\beta$ ), which then activate myeloid dendritic cells ( $\mathrm{mDCs}$ ) to produce key psoriatic inflammatory cytokines IL-12 and IL-23 [8]. mDCs can also be directly activated by nucleic acid-LL37 complexes via TLR8 [9] and then migrate to lymph nodes where they promote the differentiation and activation of T cells via TNF $\alpha$, IL-12, and IL-23. Activated $\mathrm{T}$ cells enter the circulation and move to inflamed skin via adhesion molecules on the endothelial cells of blood vessels. At the site of inflammation, different subsets of T cells release their effector molecules IFN- $\gamma$, IL-17, and IL-22. These cytokines, in concert with the pro-inflammatory signals such as IL-23 produced by $\mathrm{mDCs}$, act on keratinocytes. Keratinocytes produce AMPs, cytokines, and 
chemokines to attract other immune cells like neutrophils and macrophages (Fig. 1) [2, 5, 10].

In chronic psoriatic lesions, $\mathrm{mDC}$ produce IL-12 and IL23, which in turn constantly stimulate $\mathrm{T}$ cell subsets Th1, Th22, and Th17 to release IFN $\gamma$, IL-22, and IL-17. CD8+ T cells are also present in psoriasis, produce the same range of cytokines as CD4+ cells, and reside predominantly within the epidermis $[5,10]$.Over the last decade, it has been shown that the main source of IL-17 in psoriasis is not T cells, but rather innate cells like neutrophils and mast cells [11-14]. Neutrophils, for example, are a rich source of IL-17, AMPs, and elastase, all of which help keratinocytes hyperproliferate. Recent studies also implicate innate lymphoid cells (ILCs) as an additional source of IL-17 in psoriasis [15, 16]. IL-17 acts directly on keratinocytes, the main IL-17 receptor (IL-17R)expressing cell type, but has only limited effects on their gene expression [17]. Synergism of IL-17 and TNF $\alpha$ is key to the full-blown effect on mRNA expression of pro-psoriatic genes (such as DEFB4, S100A7, IL19, IL17C, CXCL8, CCL20, $L C N 2$ ) in keratinocytes [18]. In psoriasis, a combination of the inflammatory cytokines IL-17, TNF $\alpha$, IL-22, and IFN $\gamma$ drives keratinocyte hyperproliferation and cytokine and chemokine release [5]. Keratinocyte-produced IL-1 $\beta$ and IL-18 act on DCs and T cells, and chemokines such as CXCL-5 and CXCL-8, as well as AMPs, constantly attract neutrophils [4]. Keratinocytes also produce vascular endothelial growth factor (VEGF), which recruits and favors proliferation of endothelial cells, thereby promoting angiogenesis and creating highly vascular psoriatic plaques (Fig. 2) [10]. Together, structural abnormalities of the epidermis, inflammatory cellular skin infiltration, and increased dermal angiogenesis result in fullblown chronic psoriasis.

\section{Treatments leading to long-lasting clearance of psoriasis}

A more detailed understanding of the pathophysiology of psoriasis has revolutionized anti-psoriatic treatment and led to the successful development of various new drugs targeting key inflammatory cytokines (Table 1 ).

Nowadays, targeted therapy, in particular with antibodies against IL-17 or IL-23, permits complete clinical clearance of skin lesions in a high percentage of cases, at rates similar to those observed after PUVA treatment [31-35] but with an apparently more favorable risk profile. Severity of psoriasis is assessed with tools such as the psoriasis area and severity index (PASI), which scores both involved body area and clinical appearance of psoriatic lesions. In treated patients, complete clearance is observed in approximately 30 to $50 \%$ of patients and reduction in severity of symptoms by $90 \%$ (PASI90) in approximately 50 to $70 \%$ of patients [25, 27-29]. However, if treatment is stopped, skin lesions eventually reappear within months. Conspicuously, patients treated with psoralen plus UVA (PUVA) photochemotherapy or antiIL-23 antibodies have the longest disease-free periods. In fact, median time to recurrence was 8 months for PUVA treatment in the study by Yones et al. [32], compared to similar sustained remission times after stopping anti-IL-17 or anti-IL-23 antibody treatment. For instance, after stopping secukinumab, the
Fig. 1 Initiation phase of psoriasis. Various triggers can cause activation of keratinocytes and the release of nucleic acids and antimicrobial peptides (e.g., LL-37), which form complexes and activate plasmacytoid dendritic cells (pDCs) and myeloid dendritic cells ( $\mathrm{mDCs}$ ). DCs promote differentiation of $\mathrm{T}$ cells into Th1, Th22, and Th17 subsets. Cytokines produced by these T cells such as IFN $\gamma$, IL-17, and IL-22 act on keratinocytes and cause hyperproliferation. Keratinocytes release AMPs and chemokines and attract neutrophils and other leukocytes

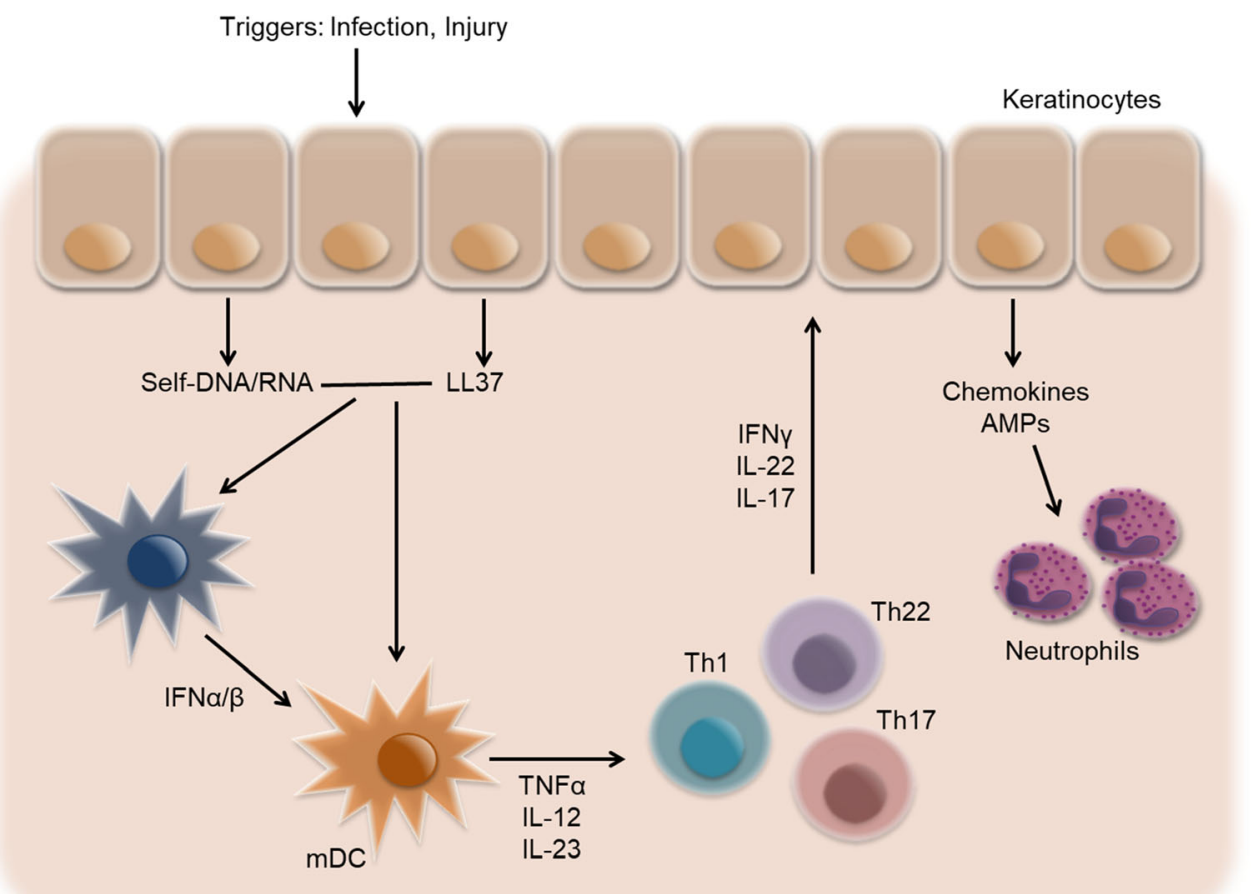


Fig. 2 Chronic psoriatic lesion. In psoriasis, mast cells, neutrophils, myeloid dendritic cells (mDCs), and $\mathrm{T}$ cells produce proinflammatory cytokines.

Proliferating keratinocytes release IL-1 $\beta$, IL-18, chemoattractants, and VEGF resulting in accumulation of neutrophils in the epidermis and increased angiogenesis

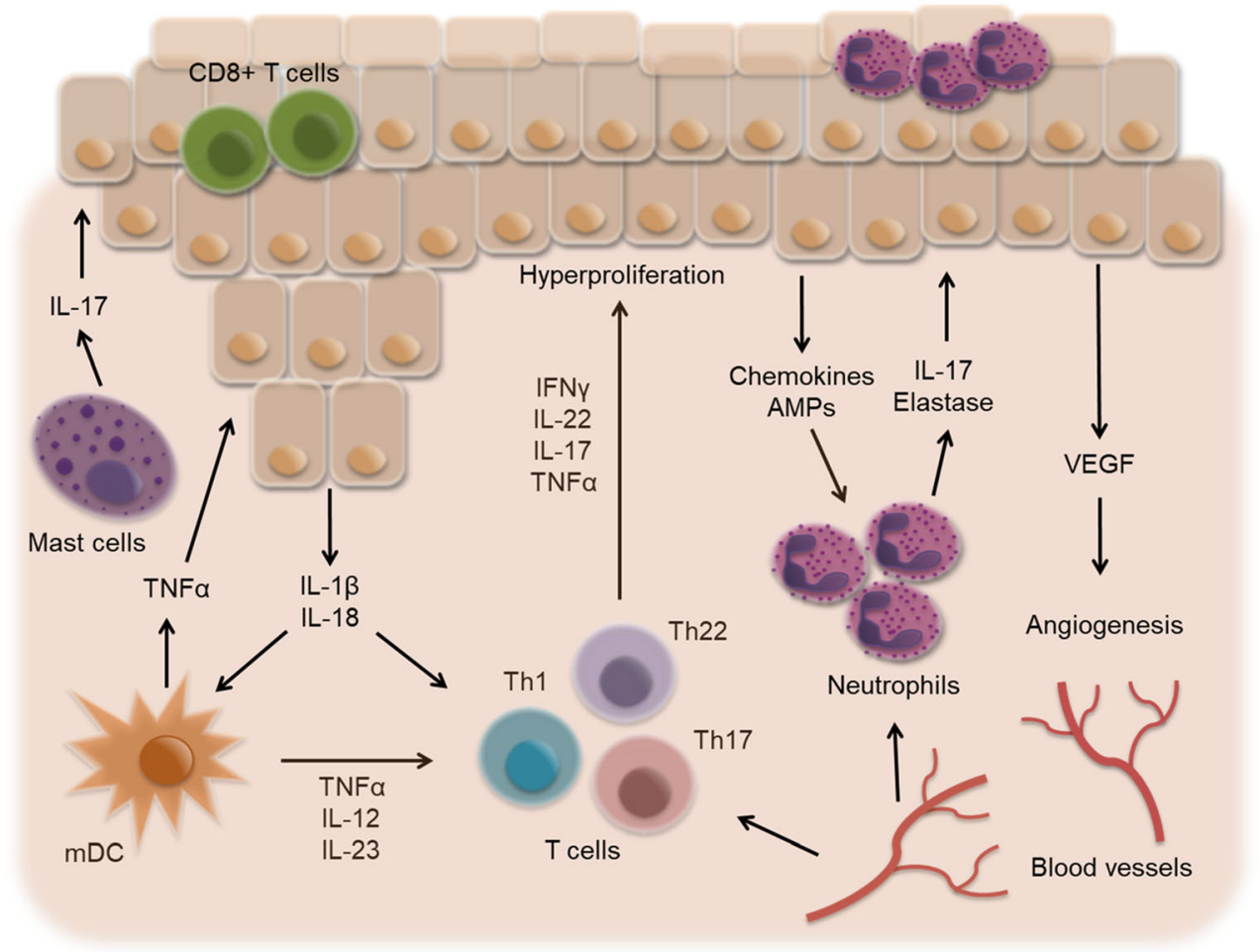

median time to relapse was 20 to 24 weeks [36]; after stopping ixekizumab, 20 weeks [37]; after stopping tildrakizumab, 20 weeks (as measured by PASI75) [26]; and after stopping guselkumab, approximately 15 weeks (as measured by PASI90) [38].

As early as 1994, Vallat et al. [39] provided a possible explanation for the longer remissions obtained with phototherapy than with other treatments such as then state-of-the-art cyclosporine. They showed that bath PUVA strongly suppressed both immunological and epidermal activation in psoriasis. Keratinocyte proteins such as keratin 16, filaggrin, and involucrin that were abnormally expressed in active psoriasis were more normally expressed after PUVA treatment. PUVA strongly suppressed epidermal and dermal CD4+ and CD8+ T cell infiltration of the skin, with virtual elimination of IL-2 receptor bearing activated T cells in some patients [39]. The authors proposed that these changes could be the cellular basis for the more sustained disease remission seen after PUVA treatment versus that seen after simple immune suppression by cyclosporine. The therapeutic mechanisms of

Table 1 Currently approved biologics for the treatment of psoriasis and their PASI75 response rates

\begin{tabular}{|c|c|c|c|c|c|}
\hline Target & Name & Type & PASI75\% $(n)$ & Timepoint & Approval \\
\hline \multirow[t]{4}{*}{$\mathrm{TNF} \alpha$} & Adalimumab [19] & Monoclonal antibody & $71 \%(578$ of 814$)$ & 16 weeks & 2008 \\
\hline & Etanercept $[20,21]$ & Soluble TNF $\alpha$-receptor & $47 \%(147$ of 311$)$ & 12 weeks & 2004 \\
\hline & Infliximab [22] & Monoclonal antibody & $80 \%(242$ of 301$)$ & 10 weeks & 2006 \\
\hline & Certolizumab Pegol [23] & PEGylated Fab' fragment & $83 \%(48$ of 58$)$ & 12 weeks & 2018 \\
\hline IL-12/IL-23p40 & Ustekinumab [24] & Monoclonal antibody & $76 \%(311$ of 411$)$ & 12 weeks & 2009 \\
\hline \multirow[t]{3}{*}{ IL-23p19 } & Guselkumab [25] & Monoclonal antibody & $91 \%$ (300 of 329$)$ & 16 weeks & 2019 \\
\hline & Tildrakizumab [26] & Monoclonal antibody & $\begin{array}{l}\text { A) } 62 \%(192 \text { of } 308) \\
\text { B) } 79 \%(236 \text { of } 298)\end{array}$ & $\begin{array}{l}12 \text { weeks (A) } \\
28 \text { weeks (B) }\end{array}$ & 2018 \\
\hline & Risankizumab [27] & Monoclonal antibody & $93 \%(77$ of 83$)$ & 12 weeks & 2019 \\
\hline \multirow[t]{2}{*}{ IL-17A } & Ixekizumab [28] & Monoclonal antibody & $\begin{array}{l}\text { A: } 98.6 \%(72 \text { of } 73) \\
\text { B: } 100 \%(72)\end{array}$ & $\begin{array}{l}12 \text { weeks (A) } \\
24 \text { weeks (B) }\end{array}$ & 2016 \\
\hline & Secukinumab [29] & Monoclonal antibody & $\begin{array}{l}82 \%(200 \text { of } 245), \\
77 \%(249 \text { of } 323)\end{array}$ & 12 weeks & 2015 \\
\hline IL-17RA & Brodalumab [30] & Monoclonal antibody & $83 \%(185$ of 222$)$ & 12 weeks & 2017 \\
\hline
\end{tabular}


action of phototherapy in psoriasis have been recently reviewed, emphasizing phototherapy's dual action on both players in the disease (i.e., keratinocytes and skin-infiltrating immune cells) [40-42].

Phototherapy induces apoptosis and immunosuppression. However, it is not clear whether these two effects act simultaneously or independently [43]. In psoriasis, PUVA [44, 45] or UVB [46-48] may act by upregulating anti-inflammatory cytokines (IL-4 and IL-10) and downregulating proinflammatory cytokines (IL-8, IL-17, IL-22, IL-23, TNF $\alpha$, and IFN $\gamma$ ). In addition, UV therapy can induce regulatory $\mathrm{T}$ cells (Tregs) $[49,50]$ and Langerhans cell emigration [51, 52]. However, it has also been shown that phototherapy leads to direct apoptosis of keratinocytes. Indeed, a study using a computational model of psoriatic epidermis combined with in vivo and in vitro experimentation revealed that apoptosis occurs in both stem and transit-amplifying cells and seems to be sufficient to explain UVB-induced plaque resolution [53].

The dual action of phototherapy (and of PUVA in particular) on keratinocytes and the immune system may be responsible for the sustained resolution of psoriatic disease after cessation of treatment. Having said that, the effect of phototherapy in psoriasis is limited to the site of exposure [31, 54], which suggests a crucial direct effect on keratinocytes (i.e., apoptosis) beyond the local and systemic effects on the immune system. We speculate that a dual effect of anti-IL-17 or anti-IL-23 antibody treatment on keratinocytes and immunocytes may also be responsible for the long-lasting, sustained response seen after treatment cessation. In contrast, other anti-psoriatic treatments such as topical steroids, vitamin $\mathrm{D}_{3}$ analogs, or anthralin [55-57], systemic traditional treatments such as cyclosporine or methotrexate, and even firstgeneration biologics (i.e., TNF antagonists) are usually associated with rather quick relapses within several weeks after treatment is stopped.

\section{Resolution of psoriasis: remaining molecular imprints}

Nowadays, psoriasis is considered as systemic disease often associated with comorbidities such as metabolic syndrome, hypertension, elevated blood lipid levels, and cardiovascular disease [58]. Intriguingly, psoriatic plaques appear in most patients only at certain body locations, in particular at predilection sites such as the extensor surfaces of the extremities (knee and elbows) and/or other mechanically stressed locations such as the sacral region. Even more intriguingly, despite a general genetic predisposition, more than 35 psoriatic susceptibility genes are known so far [59], certain patients develop life-long psoriasis only on fixed, circumscribed, and sometimes solitary body sites. Psoriatic lesions can resolve entirely after treatment and always without scarring. However, when treatment is stopped, lesions recur most often at the exact same body sites. This raises an important question: what kind of residual "molecular scars" are left behind on a clinically cleared psoriatic lesion that eventually cause its reappearance. What those molecular scars look like after treatment with topical and traditional systemic agents is obscured by insufficient data, but what they look like after treatment with anti-TNF $\alpha$, anti-IL-17, and anti-IL-23 antibodies is clearer thanks to recent studies of skin biopsy samples.

\section{Anti-TNFa treatment}

As early as 2007, when the crucial role of Th17 cells in psoriasis was not fully appreciated, Zaba and colleagues studied the effects of the soluble TNF $\alpha$ receptor fusion protein etanercept in a clinical trial enrolling 20 patients at several time points of biopsy sampling during 12 weeks of treatment [60]. Responders and non-responders were grouped with regard to remaining epidermal thickness and normalization of keratinocyte hyperproliferation as measured by Ki67 and K16 protein and KRT16 gene expression. Fast normalization of keratinocyte hyperproliferation along with reduced expression of Th17-related genes (IL17, CCL20, DEFB4) was observed after only 1 week of TNF $\alpha$ inhibition. In addition, inflammatory products of DCs (INOS, TNF, IL20, IL23p40) were reduced early in treated psoriatic plaques. Going along with a significantly reduced PASI score, there were diminished numbers of dendritic cells and T cells and macrophages as well as a repressed IL22 mRNA expression in psoriatic plaques after 2 weeks of etanercept treatment. Interestingly enough, Zaba et al. found a rather delayed response of Th1-related genes (IFNG, LTA1). They therefore proposed a model mechanism of TNF $\alpha$ inhibition in psoriasis where inflammatory DCs are quickly inhibited, in turn repressing Th17 response and keratinocyte hyperproliferation. They also argued that a delayed effect on Th1 response is still needed to completely clear psoriasis [60]. In 2009, the same group of investigators published a comprehensive microarray study, extending their previous findings. Intriguingly, immediate response genes of $\mathrm{TNF} \alpha$ inhibition were linked to myeloid cells (DCs, Langerhans cells), but not $\mathrm{T}$ cells. Resident DC genes were upregulated early during treatment (myeloid DC genes $C D 1 C$ and $C D 1 E$ and Langerhans cell genes CD207 and CD1A), with CD1c and CD207 cell counts supporting these results by being low at baseline, increasing at week 2 , and then decreasing at week 12 to baseline levels. Furthermore, they saw a rapid decrease of TNF-response genes $I L 1 B$ and CXCL8 in both responders and non-responders. At the late time point, $\mathrm{TNF} \alpha$ and IFN $\gamma$ pathway genes were downregulated in both responders and non-responders, but IL-17 pathway genes were only downregulated in responders. This led Zaba et al. to conclude that suppression of Th17 response genes is necessary for resolution of psoriatic disease [61]. 
In 2011, Suárez-Fariñas and colleagues [62] were the first to define the term "residual disease genomic profile" in psoriasis. They analyzed biopsy samples from clinical and histological responders to etanercept. Histologically, sampled skin was classified as "normal", as epidermal thickness had improved by nearly $100 \%$ and CD3+ T cells, DCs and macrophage cell counts had returned to non-lesional levels. The exception was CD8+ T cell infiltration of the skin, which was only partially reduced (by 64\%) after etanercept treatment. For transcriptional analyses, the expression of known psoriasis-associated genes in pretreatment lesions was compared with those in resolved lesions. Not surprisingly, the expression of many inflammatory and keratinocyte-related genes had improved by more than $90 \%$. Although upregulation of $\mathrm{T}$ cell genes was reduced by $88 \%$, that of important inflammatory genes like IL22, IL 17 , $I L 12 p 35$, and $I F N G$ was reduced to a lesser extent (by approximately $65 \%$ ). A "residual disease genomic profile" was defined as psoriasis-associated genes improving less than $75 \%$. This profile was composed of about 250 transcripts and was divided into two functional groups, inflammation-associated genes and structural genes (e.g., genes related to lymphatic endothelial cells). These findings suggested that inflammation does not completely resolve even when psoriatic lesions are clinically healed and that some residual CD8+ T cells remain in the skin. In addition, some structural abnormalities of the skin were not fully reversed.

In 2014, Johnston et al. investigated very early changes in lesional skin during TNF inhibition by etanercept [63]. In their study, 20 etanercept responders were studied and gene expression was analyzed using qPCR and microarray at baseline, after 1 day, 3 days, 7 days, and 2 weeks of TNF inhibition. There were no changes in mRNA expression of IL17A, IL22, and $I F N G$ in the first week of therapy. In line with the findings of Zaba et al. [61], other IL-17 pathway-related genes were suppressed by etanercept. Interestingly, Johnston and colleagues were able to show downregulation of IL-17 receptor $\mathrm{C}(I L 17 R C)$ after only 1 day of TNF $\alpha$ inhibition. Next, they compared microarray results in their etanercept-treated patients with those in ixekizumab (anti-IL-17A)-treated patients and found an extensive overlap in downregulated genes. When normal human keratinocytes were treated with $\mathrm{TNF} \alpha$ in vitro, mRNA and protein expression of $I L 17 R C$ increased. By inhibiting $I L 17 R C$ using short hairpin RNA (shRNA), effects of TNF $\alpha$ were suppressed, indicating that etanercept acted by blocking IL-17A signaling. However, etanercept obviously does not directly target Th17 cytokines because IL17A, IL22, and activated STAT3 expression levels were still high in treated lesions and were downregulated only much later. Early effects of TNF $\alpha$ inhibition might include suppression of IL-17RC in keratinocytes, in turn leading to decreased IL-17A sensitivity in the tissue and thereby halting the inflammatory feedback loop [63]. Similar results were found by investigating the effects of the TNF $\alpha$ antibody adalimumab.
Genes associated with keratinocyte hyperproliferation were normalized, and mRNA expression of Th17-associated cytokines was downregulated [64, 65]. Moreover, Bose et al. [66] reported from a study of the effects of etanercept, adalimumab, and infliximab on psoriatic lesions that the anti-TNF-modulated genes most closely associated with clinical improvement were those encoding CCR-7 and its ligand, CCL-19, as well as genes involved in dendritic cell maturation, $\mathrm{T}$ cell activation, and VEGF expression.

\section{Anti-IL-17 treatment}

Currently, two IL-17A antagonists are available for the treatment of psoriasis: ixekizumab (approved in 2016) and secukinumab (approved in 2015). In 2012, when the role of $\mathrm{T}$ cell subsets in psoriasis was not yet fully defined, Krueger and colleagues hypothesized that Th17 cells might be crucial and studied effects of IL-17 inhibition using the anti-IL-17A antibody ixekizumab. As they demonstrated by immunohistochemistry, ixekizumab not only decreased keratinocyte hyperproliferation (K16, Ki-67) and skin infiltration by T cells (CD3) and dendritic cells (CD11c), but also suppressed release of keratinocyte-produced AMPs (LL-37, S100A7, S100A8, and BD2). They next investigated early changes in gene expression in the skin during IL-17 inhibition using qPCR. After 2 weeks, expression of KRT16, IL17A, IL17F, IL22, IL23p19, and $L C N$ was decreased, and microarray analysis showed that 765 genes were already differentially expressed compared to baseline. Among them were IL19 (inducer of epidermal hyperplasia); CXCL8 and CXCL1 (IL-17induced neutrophil chemokines); CCL20 (chemokine for Th7 cells and dendritic cells); GZMB (granzyme B; effector molecule of cytotoxic T cells); and $L C N$ (IL-17-induced AMP). Next, transcriptional data on skin samples from ixekizumabtreated patients were compared to etanercept's. Of 1200 psoriasis genes, about 600 were normalized at 2 weeks by ixekizumab, but only about 100 were normalized by etanercept, translating into an improvement of $70 \%$ with IL17 inhibition versus $35 \%$ with TNF inhibition. Interestingly, IL-17 pathway-associated molecules such as $C X C L 1, C X C L 8$, $D E F B 4$, and $L C N$ correlated with improvement of psoriatic epidermis at week 2 , but $I L 17 A$ and $I L 17 F$ did not. Furthermore, epidermal improvement was not linked to suppression of Th1- and Th22-associated genes. This led Krueger et al. to conclude that early improvement during ixekizumab therapy is linked to suppressing IL-17's effect on keratinocytes and not to changes in $\mathrm{T}$ cell infiltration of the skin or cytokines produced by T cells [67].

In the last few years, evidence has accumulated that $\mathrm{T}$ cells are not the only cell type producing IL-17 in psoriasis [11-13][14]. To further investigate the various sources of IL17, Reich et al. [68] analyzed effects of IL-17 inhibition in secukinumab-treated patients after 2 and 12 weeks of therapy. 
After 2 weeks, neutrophil counts in the skin and markers for epidermal hyperproliferation were reduced, accompanied by a reduction of expression of IL-17-induced neutrophil chemoattractants (CXCL1, CXCL8), which are produced by keratinocytes. A significant decrease in mRNA expression was also observed for $I L 17 A$ and $I L 17 F$, but not for $T N F$. Interestingly, $\mathrm{T}$ cell and dendritic cell infiltration of the skin decreased more slowly. Immunostaining revealed that neutrophils were the predominant IL-17 source, followed by T cells and mast cells. However, mast cell numbers in the skin remained unchanged during and after secukinumab treatment. In comparison, the clinical result in a low-dose group in the same study was worse at 12 weeks and, strikingly, neutrophil counts and neutrophil chemoattractants were increased again. In general, patients with detectable neutrophil infiltration at 12 weeks had a shorter time to relapse than those who did not. These results led to the conclusion that neutrophils are an important source of IL-17 in psoriasis and that secukinumab suppresses crosstalk between keratinocytes and neutrophils. In this crosstalk, IL-17 produced and/or stored by neutrophils, mast cells, and T cells stimulates epidermal cells to produce neutrophil chemoattractants, leading to increased neutrophil counts in psoriasis. Inhibition of this crosstalk could be an early mechanism of secukinumab, while full clinical effects are linked to a decrease in T cells and dendritic cells in the skin [68]. These molecular results of IL-17A inhibition through secukinumab were further elucidated in 2017 by Kolbinger and colleagues [69], who looked at protein levels and gene expression in the skin of eight patients treated with secukinumab. Among the top 10 downregulated proteins after 8 and 15 days were IL- $1 \beta$, AMPs ( $\beta$-defensin 2 and LCN2), neutrophil enzyme MPO, neutrophil chemoattractants (CXCL-1 and CXCL-5), and Th17 chemoattractant CCL-20. Early downregulation of gene expression was observed in AMPs (BD2, LCN2, LL37, S100A8, and S100A9) as well as neutrophil- and Th17-attracting chemokines (CXCL1, CXCL8, and CCL20) and the IL-1 family member IL36A. Serum protein levels of IL-17A and $\beta$-defensin 2 correlated with PASI reduction in the treated patients, suggesting that serum $\beta$-defensin 2 in particular could be a potential biomarker for response to secukinumab [69].

Brodalumab is an IL-17RA-targeting monoclonal antibody that has shown high clinical efficacy [30]. Transcriptional analysis of psoriatic skin samples conducted by Russel et al. [70] revealed that brodalumab, similar to other biologics, induces early changes in keratinocyte markers after IL-17RA inhibition, accompanied by a delayed response of $\mathrm{T}$ cell and leukocyte genes. Within 2 weeks after start of treatment, keratinocyte genes such as IL36A, S100A7, KRT6, CXCL6, and $I L 17 C$ had decreased markedly, and certain inflammatory cytokines such as IL12A and IL23A were normalized, while the expression of $I L 17 A, I L 17 F$, and IL22 was only partially reduced and decreased more substantially after 6 weeks. Also, as in other studies with biologics, epidermal proliferation was reduced quickly, followed by a slow decrease in T cell counts in the skin and complete normalization at 6 weeks after start of brodalumab treatment. In addition, the investigators generated and compared gene expression scores for keratinocyte genes induced by either IL-17 or IFN $\gamma$ during the course of brodalumab treatment. While the IL-17 score almost returned to levels seen in non-lesional skin, the IFN $\gamma$ score only partially diminished. Clinical improvement of psoriasis correlated with normalization in the expression of IL-17-induced keratinocyte genes. Thus, IL-17R blockade by brodalumab may directly target keratinocytes, since there is a fast normalization of keratinocyte markers and slower changes in cytokines produced by T cells, as well as T cell numbers in the skin after treatment. The slow decrease of IL-22 and remaining high levels of IFN $\gamma$ imply that clinical and molecular improvement through brodalumab treatment depends little on changes in Th1 and Th22 cells [70].

\section{Anti-IL-23 treatment}

The cytokines IL-12 and IL-23 are both heterodimers, sharing the IL-12/IL-23p40 subunit, and additionally consist of IL-12p35 and IL-23p19, respectively. Both cytokines are produced by myeloid dendritic cells and influence $\mathrm{T}$ cell differentiation [71]. Since IL-23 helps to drive psoriasis, several biologics have been designed that target its subunits, p40 (targeted by ustekinumab) and p19 (targeted by guselkumab, risankizumab, and tildrakizumab). Brodmerkel et al. [72] compared global mRNA expression changes by microarray analysis in psoriatic skin samples from patients treated and achieving PASI75 with the anti-IL-12/IL-23p40 drug ustekinumab versus with etanercept. Within 12 weeks, both ustekinumab and etanercept caused a significant change in 5000 genes and 4500 genes, respectively. While the majority of differentially expressed genes (DEGs) were shared by both drugs, inhibition of IL-12/IL-23p40 uniquely modulated 700 genes and suppression of TNF $\alpha$ uniquely altered expression of around 400 other transcripts. Looking more closely at prominent psoriasis genes, a strong downregulation of IL-17related genes (IL19, DEFB4, CCL20, LCN2, IL1B) and a less impressive but still significant reduction of IL-17A and IL-23 subunit genes were observed after treatment with both drugs. However, overall psoriasis-associated genes and genes belonging to signaling pathways of IL-22, IFN $\gamma$, TNF $\alpha$, IL1, and IL-17 were more strongly suppressed by ustekinumab than by etanercept. While a stronger effect on IFN $\gamma$ associated genes by IL-12/IL-23p40 inhibition was anticipated, the strong impact on TNF-associated genes was surprising.

The term "molecular scar" has been used for residual disease genes that are less than $75 \%$ suppressed from baseline levels by treatment [62]. After ustekinumab treatment, the expression 
levels of $18 \%$ of disease-related genes did not return to baseline levels compared with $23 \%$ after etanercept at similar clinical efficacy. This led Brodmerkel et al. to conclude that the smaller molecular scar left after ustekinumab treatment may create a more stable environment for resolved lesions, resulting in a lower likelihood of disease recurrence due to reduced IL-17 expression by T cells induced by blockade of IL-23. Etanercept, on the other hand, blocks IL-23 only indirectly, as it targets IL-23-producing dendritic cells by diminishing the action of TNF $\alpha$ [72] and thus may have a weaker effect on IL-17 levels.

Visvanathan and colleagues compared the molecular scar left after treatment with the IL-23p19 inhibitor risankizumab versus with ustekinumab [73]. Clinically, risankizumab is overall more effective than ustekinumab [27, 73]. To determine why, cellular markers and gene expression profiles were compared in skin samples from patients treated with the drugs. Both drugs lessened epidermal thickness and reduced protein levels of K16, CD3, CD11c, DC-LAMP, Ki67, S100A7, LCN2, and $\beta$-defensin-2 after 4 weeks. Transcriptional analyses showed that both biologics led to early decreases of IL-17/IL-23 pathway genes (e.g., IL17A, IL17F, IL17C, IL22, IL23A, S100A8, S100A9, LCN2, and BD2). Risankizumab, however, had a stronger decreasing effect on genes that were upregulated in reconstructed epidermal cells and keratinocytes after in vitro stimulation with IL-17 [73]. Differences were also observed in psoriasis-associated genes related to keratinocytes, monocytes, and macrophages. Overall, risankizumab had a stronger effect on disease-related genes than ustekinumab did, although PASI reduction after 4 weeks was similar [73].

Guselkumab is another antibody against IL-23p19 approved for treatment of psoriasis. In 2014, Sofen et al. [74] analyzed effects of IL-23p19 inhibition in 24 psoriasis patients after 1 and 12 weeks of treatment. After 1 week, only slight changes in epidermal thickness and CD3 and CD11c positive cells were found. After 12 weeks, epidermal thickness as well as protein expression of keratin 16 and CD3 and CD11c cell counts in the skin were significantly reduced. A significant reduction of epidermal hyperplasia gene KRT16 and IL-17 pathway-related genes $S 100 A 7, L C N 2, C X C L 1$, and CXCL8 and a modest reduction of IL17A were established by qPCR. Genome-wide transcriptional analysis revealed normalization by $70 \%$ or more of almost all disease-related genes (about 1200 genes) after 12 weeks of guselkumab treatment. In fact, the mRNA profile after 12 weeks of IL-23p19 inhibition closely resembled that of nonlesional skin. To further extend their findings, Sofen et al. performed serum immunoassays. Interestingly enough, protein levels of IL-17A were already strongly reduced after 1 week and then further decreased at 12 weeks. However, guselkumab did not affect serum levels of various other psoriasis-related cytokines, including IL-23p19 [74].

In sum, blocking solely the p19 subunit of IL-23 and not both p19 and p40 is a more effective approach in the treatment of psoriasis. Aside from neutralizing IL-23, which is thought to be responsible for the curative effect, anti-p40 therapy also interferes with IL-12 signaling and type 1 immunity [75]. Using a preclinical model for psoriatic plaque formation Kulig et al. [75] showed that IL-12, in contrast to IL-23, had a regulatory function by restraining the invasion of an $\mathrm{IL}-17$-committed $\gamma \delta \mathrm{T}(\gamma \delta \mathrm{T} 17)$ cell subset and that IL-12 receptor signaling in keratinocytes initiated a protective transcriptional program that limited skin inflammation. Thus, therapeutic collateral targeting of IL-12 and IL-23 may be counterproductive in the therapy of psoriasis. Indeed, the findings by Kulig et al. help to explain the therapeutic inferiority of IL-12/23 inhibition by ustekinumab to pure anti-IL23 inhibition by risankizumab, guselkumab, or tildrakizumab.

\section{Cellular scar}

The fact that psoriasis recurs most often at the very same body sites where the initial lesion occurred suggests that critical cells may be left behind in the skin in situ after treatment is stopped. In this regard, Cheuk and coworkers [76] reported the results of an elegant study in 2014, addressing the question whether psoriatic lesions harbor tissue-resident memory T cells (TRMs) that could possibly drive disease recurrence at the same, i.e., initial site of lesions. They sampled narrow band-UVB-treated, anti-TNF $\alpha$ or anti-IL-12/23-treated healed psoriatic skin and studied gene expression and ex vivo cytokine production by $\mathrm{T}$ cells. Interestingly, they found that approximately half of CD8+ T cells in the epidermis of active lesions expressed TRM markers CD103 and CD49a. This suggested an expansion of TRMs at diseased body sites of psoriasis compared with healthy skin. In resolved lesions, epidermal CD4+ T cells had returned to normal levels, while numbers of dermal CD4+ and CD8+ T cells were still elevated. Although epidermal CD8+ T cells were decreased, the number of CD49a-expressing CD8+ T cells was still higher than in non-lesional or healthy skin (that had not had visible psoriatic manifestation). Transcriptional analysis of isolated $\mathrm{T}$ cells from resolved psoriatic lesions showed that epidermal $\mathrm{T}$ cells had higher expression of Th17-associated genes (RORC, $I L 17 A, I L 22)$ than did dermal T cells. Upon ex vivo stimulation, dermal $\mathrm{T}$ cells from those lesions had a cytokine production profile similar to that found in healthy skin. Interestingly, the percentage of IL-22+ epidermal CD4+ T cells from resolved lesions was similar to that in active lesions. Epidermal CD8+ T cells from resolved lesions produced more IL-17A after ex vivo stimulation than did those from healthy skin, indicating that these cells are still functional even after long-term anti-psoriatic treatment. Epidermal IL-17-producing CD8+ T cells from healthy skin, lesional psoriasis, and resolved psoriasis expressed CD103, suggesting that these are in fact TRMs. In resolved lesions, an epidermal subpopulation of CD8+ T cells coexpressed TRM marker CD103 and Th17 markers IL-23R and CCR6, indicating responsiveness to IL-23 signaling. Moreover, Cheuk et al. [77] found two subsets of CD8+ TRMs in the 
epidermis, IL-17-producing CD49- and IFN $\gamma$-producing CD49+ TRMs. Intriguingly, IL-17-producing CD49- TRMs from the epidermis showed higher IL-17 production than did CD49TRMs isolated from dermis. This suggested that distinct functional subsets exist in epidermis and in dermis. In active psoriasis, some CD49- TRMs as well as some CD49+ TRMs expressed both IL-17 and IFN $\gamma$. However, after isolation and ex vivo stimulation, these cells did not produce IL-17. Thus, the investigators speculated that $\mathrm{T}$ cell receptor (TCR) activation might be needed in addition to inflammatory signals promoting IL-17 response [77].

Most recently, Matos and co-workers [78] used immunofluorescence staining and high-throughput sequencing (HTS) of the CDR3 domain of the $\mathrm{T}$ cell receptor as a different approach to assess density and clonality of T cells in order to study residual $\mathrm{T}$ cells in resolved psoriatic lesions. TCR sequencing indicated that there were more $\mathrm{T}$ cells in resolved psoriatic lesions than in the skin of healthy controls and non-lesional skin of psoriasis patients; in fact, levels in resolved lesions were similar to those in active lesions. While diverse $\mathrm{T}$ cell clones were present in active lesions before treatment, only $7 \%$ of $\mathrm{T}$ cell clones remained detectable in clinically healed lesions. This suggests that, when inflammation subsides, the majority of $\mathrm{T}$ cell clones leave the skin. Increased numbers of oligoclonal $\mathrm{T}$ cell populations were found not only in resolved lesions but also in non-lesional skin. When $\mathrm{T}$ cells were isolated from resolved lesions to study their cytokine production ex vivo, expanded $\mathrm{T}$ cell clones from both active and clinically healed lesions produced IL-17A. TCR sequencing also showed that putative pathogenic $T$ cell clones were $\alpha \beta$ T cells (implying that their TCR is composed of $\alpha$ - and $\beta$ chains) and not $\gamma \delta$ T cells. Furthermore, unique $\alpha \beta$ TCR sequences that were identified in the skin of psoriasis patients were absent from the skin of healthy controls and patients with other skin diseases. These findings implied that, in psoriasis, increased $\mathrm{T}$ cell response might be due to the presence of a common antigen. Pathogenic T cell clones producing IL-17A and residing in low numbers in clinically healed lesions could in fact be TRMs. They may be resistant to elimination by therapy and bear the potential to restart the psoriatic inflammatory feedback loop at the site of residence [78].

In 2018, Gallais-Sérézal and colleagues studied how resolved psoriatic tissue responded to activation of putative pathogenic TRMs. Using sampled skin biopsies from healthy controls and resolved lesions from psoriasis patients, they produced skin explant cultures and then stimulated them with anti-CD3 antibody OKT-3 to activate resident T cells. The skin environment after $\mathrm{T}$ cell activation was analyzed by NanoString transcriptional methodology, and IFN $\gamma$-related signaling pathways were found to be upregulated in both healthy and resolved psoriatic skin. However, in resolved psoriasis alone (and not in healthy skin), IL-17 signaling was upregulated in the epidermal compartment. This implied that renewed activation of resident $\mathrm{T}$ cells in resolved lesions leads to keratinocyte activation, which could translate into chemokine release and recruitment of circulating leukocytes. Indeed, IL-17 signaling-related tissue-response correlated with clinical relapse after therapy, thus highlighting the key role of pathogenic resident $\mathrm{T}$ cells in psoriasis [79]. The same group of investigators studied TRMs in non-lesional skin from psoriasis patients to determine whether these resident $\mathrm{T}$ cells are indeed pathogenic and have the ability to induce psoriasis in never-lesional skin. Indeed, ex vivo T cell activation of skin explants led to psoriasis-typical tissue responses as determined by NanoString analysis. Additionally, CD103+ CD8+ $\mathrm{T}$ cells and CCR6+ CD4+ T cells were enriched in nonlesional skin of psoriasis patients compared with that in healthy controls. Moreover, CCR6+ CD4+ T cells were capable of producing IFN $\gamma$ and IL-17. These results suggested that pathogenic TRMs do exist in non-lesional skin [80].

Although pathogenic TRMs have certainly gained more attention in recent years, some progress has been made in analyzing the presence and function of DCs and Langerhans cells in resolved psoriasis as well. While DC numbers were found to be high in active psoriatic lesions and absent from resolved skin, the data on Langerhans cells are controversial [81, 82]. Langerhans cells from active lesions expressed psoriasis-associated genes $(I L 23, I L 1 B, I L 15)$ and showed increased IL-23 production after ex vivo stimulation. In resolved lesions, expression of IL23 and IL15 was still higher than in healthy skin. Upon ex vivo stimulation, these cells were still capable of producing IL-23 [83]. In a comprehensive review on the role of Langerhans cells in psoriasis, Eidsmo and Martini [81] hypothesized that Langerhans cells are not only found in close proximity to $\mathrm{T}$ cells, but are also able to cross-talk with both $\mathrm{T}$ cells and keratinocytes. This, together with the ability of Langerhans cells to produce IL-23, implies that Langerhans cells could play a role in renewed activation of $\mathrm{T}$ cells and disease recurrence.

Last but not least, Johnson-Huang and colleagues [46] showed that NB-UVB leads to clearance of psoriasis by suppressing the IL-17/IL-23 axis. They compared responders vs. non-responders after 6 weeks of NB-UVB therapy and looked at myeloid DCs and $\mathrm{T}$ cells and their inflammatory products using immunohistochemistry and qPCR. Inflammatory myeloid DC levels were reduced and expression of DC-related cytokines (e.g., INOS, IL20, IL23p19, IL12/IL23p40) was decreased in responsive plaques. Furthermore, CD3 T cell counts were reduced in responders, and cell counts strongly correlated with the defined "response score". In addition, expression of $I F N G$, IL17, IL22, and IL-17/IL-22 downstream genes $\beta$-defensin 4 (BD4) and myxovirus-resistance $1(M X 1)$ was significantly suppressed. All of these changes were not observed in nonresponsive plaques. Interestingly, the "response score" correlated with $I L 17$ and IL22 expression, but not with $I F N G$ expression. To determine whether these results show a direct effect of NBUVB or merely changes that occur when psoriasis clears up in 
general, Johnson-Huang's group performed an in vitro experiment where PBMCs were isolated, treated with NB-UVB, and measured for cytokine expression after $4 \mathrm{~h}$. Intracellular staining showed that production of IFN $\gamma$ was suppressed by $85 \%$ and that IL-17 and IL-22 produced by CD3+ T cells were decreased by $45 \%$ and $89 \%$, respectively [46]. It is fascinating to learn that a nature-derived therapy such as UVB phototherapy seems to interfere with the very same pathway targeted by today's most modern drug treatments, i.e., the IL-17/23 axis.

\section{Conclusion and open questions}

Numerous studies have shown that the molecular imprints of psoriasis do not fully resolve in macroscopically cleared skin even after the most efficacious targeted treatment available nowadays, including anti-IL-17 and anti-IL-23 antibody administration. Resolved lesions have a molecular scar composed of about 250 gene products that are not fully normalized after treatment. These transcripts can be grouped into inflammation-associated genes such as IL17, IL22 and others and skin structure-related genes [62]. Moreover, pathogenic memory $\mathrm{T}$ cells seem to persist at sites of clinically resolved psoriatic lesions. Even after long-term therapy, these cells do not lose their ability to produce IL-17A [76, 78], which can signal to keratinocytes and stimulate their proliferation [80]. Although the role of innate cells has not been studied extensively, Langerhans cells isolated from resolved psoriatic lesions can produce IL-23 after stimulation, which makes them another potential player in the restart of activation of "sleeping" TRMs [83] and possible reappearance of psoriatic lesions [76] (Fig. 3). The question now is this: what initially triggers (or retriggers) the process of psoriatic recurrence after a treatment has been stopped? Could IL-17 be stored and hidden in certain cells of the skin, perhaps mast cells, waiting to be released after endogenous and/or exogenous stimulation to activate the inflammatory loop again? What about the neurogenic inflammation of the skin [84] that plays an essential role at least in the itch of psoriasis? Might a nerve scar left behind after successful treatment contribute to the reappearance of psoriatic lesions, be it during or after continuous treatment? Last but not the least, may the interplay of the microbiome and AMPs [85, 86] help trigger psoriatic recurrence? This may be in particular important for pustular psoriasis, in which IL-1/IL-36 plays an important role in the pathogenesis [87-89]. A vicious loop between AMPs such as cathelicidin (LL-37) and IL-36 signaling may drive psoriatic disease [90-93]. In fact, IL-36 receptor blockade revealed promising results in pustular psoriasis [94] and may also be a therapeutic option in plaque-type psoriasis [95]. Future work will have to address all of these open questions in order to improve and advance overall treatment strategies for psoriasis and allow long-term, continuous disease control.
Fig. 3 Model mechanism for disease recurrence in resolved lesions. In clinically healed lesions, CD4+ T cells remain in the dermis and express IL-22 mRNA. Langerhans cells (LCs) residing in the epidermis express IL-23 mRNA. Epidermal CD8+ TRMs expressing $\alpha \beta$ TCR are able to produce IL-17. Upon disease trigger, LCs and T cells actively produce proinflammatory cytokines and cause recurrent inflammation

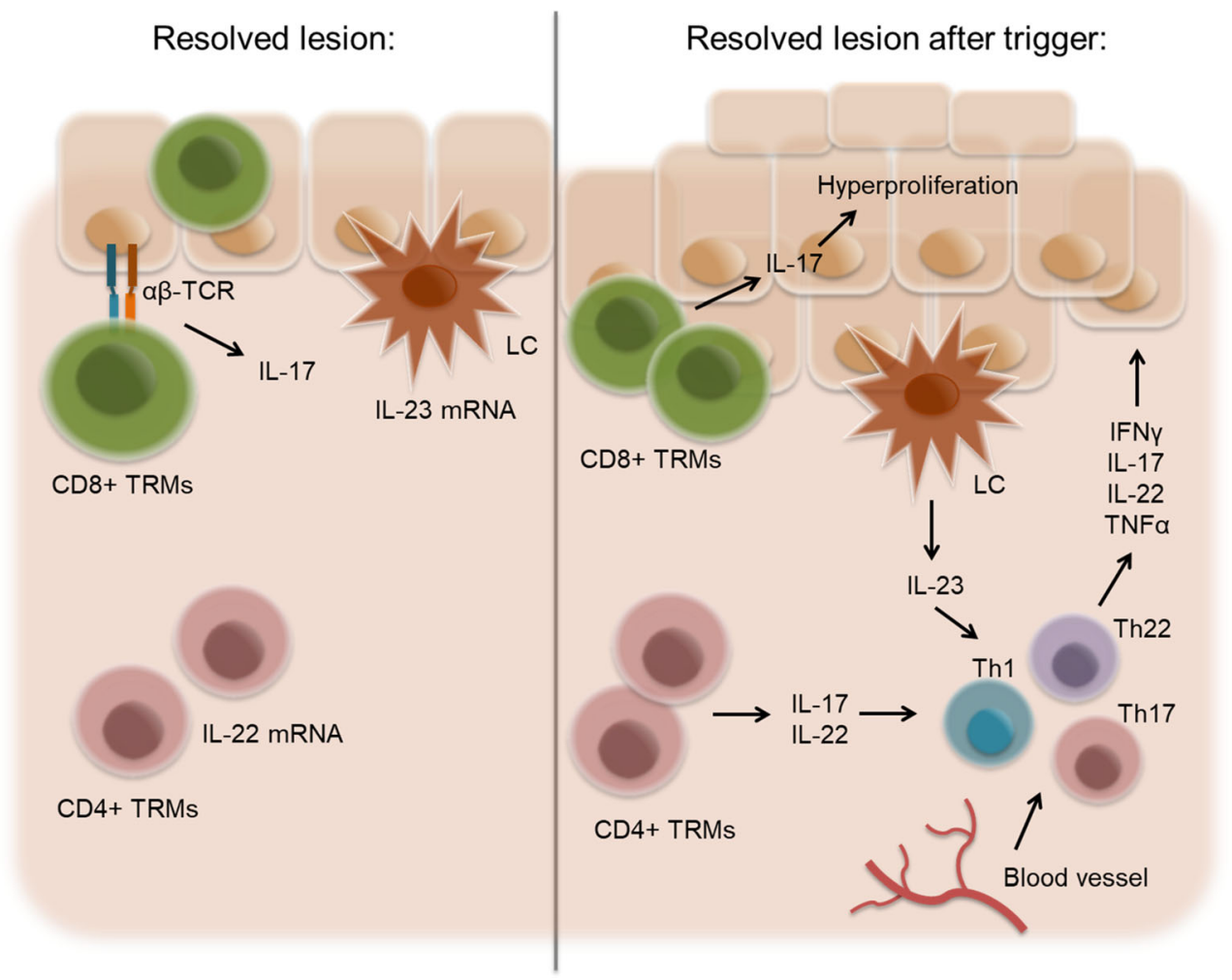


Acknowledgments The authors would like to thank Dr. Honnovara N. Ananthaswamy, Houston, TX, for critical reading and Jude Richard, Austin, TX, for editing the manuscript.

Funding information Open access funding provided by Medical University of Graz. The authors received support from the Austrian Science Fund FWF (W1241) and the Medical University of Graz through the PhD Program Molecular Fundamentals of Inflammation (DKMOLIN).

\section{Compliance with ethical standards}

Conflict of interest PW has been a consultant, lecturer or investigator for AbbVie, Almirall, Amgen, Celgene, Eli Lilly, Leo Pharma, JanssenCilag, Leo Pharma, Merck Sharp \& Dohme, Sandoz, Sanofi-Aventis, UCB Pharma, and Pfizer.

Open Access This article is distributed under the terms of the Creative Commons Attribution 4.0 International License (http:// creativecommons.org/licenses/by/4.0/), which permits unrestricted use, distribution, and reproduction in any medium, provided you give appropriate credit to the original author(s) and the source, provide a link to the Creative Commons license, and indicate if changes were made.

\section{References}

1. Di Meglio P, Villanova F, Nestle FO (2014) Psoriasis. Cold Spring Harb Perspect Med 4(8):1-30

2. Lowes MA, Bowcock AM, Krueger JG (2007) Pathogenesis and therapy of psoriasis. Nature 445(February):866-873

3. Michalek IM, Loring B, John SM (2017) A systematic review of worldwide epidemiology of psoriasis. J Eur Acad Dermatol Venereol 31:205-212

4. Nestle FO, Kaplan DH, Barker J (2009) Psoriasis. N Engl J Med 361(5):496-509

5. Lowes MA, Suarez-Farinas M, Krueger JG (2014) Immunology of Psoriasis. Annu Rev Immunol 32:227-255

6. De Rosa G, Mignogna C (2007) The histopathology of psoriasis. Reumatismo 59:46-48

7. Lande $\mathrm{R}$ et al (2007) Plasmacytoid dendritic cells sense self-DNA coupled with antimicrobial peptide. Nature 449:564-571

8. Lande R et al (2014) The antimicrobial peptide LL37 is a T-cell autoantigen in psoriasis. Nat Commun 5(5621):1-15

9. Ganguly D et al (2009) Self-RNA - antimicrobial peptide complexes activate human dendritic cells through TLR7 and TLR8. J Exp Med 206(9):1983-1994

10. Mahil SK, Capon F, Barker JN (2016) Update on psoriasis immunopathogenesis and targeted immunotherapy. Semin Immunopathol 38(1):11-27

11. Lin AM et al (2011) Mast Cells and Neutrophils Release IL-17 through Extracellular Trap Formation in Psoriasis. J Immunol 187:490-500

12. Brembilla NC, Stalder R, Senra L, Boehncke WH (2017) IL-17A localizes in the exocytic compartment of mast cells in psoriatic skin. Br J Dermatol 177:1458-1460

13. Dyring-Andersen B et al (2017) Interleukin (IL)-17A and IL-22producing neutrophils in psoriatic skin. Br J Dermatol 177:321-322

14. Patel DD, Lee DM, Kolbinger F, Antoni C (2013) Effect of IL-17A blockade with secukinumab in autoimmune diseases. Ann Rheum Dis 72:1-8.
15. Keijsers RRMC, Joosten I, van Erp PEJ, Koenen HJPM, van de Kerkhof PCM (2014) Cellular sources of IL-17 in psoriasis: A paradigm shift? Exp Dermatol 23(11):799-803

16. Keren A et al (2018) Innate lymphoid cells 3 induce psoriasis in xenotransplanted healthy human skin. J Allergy Clin Immunol 142(1):305-308

17. Nograles KE et al (2008) Th17 cytokines interleukin (IL)-17 and IL-22 modulate distinct inflammatory and keratinocyte-response pathways. Br J Dermatol 159(5):1092-1102

18. Chiricozzi A et al (2011) Integrative responses to IL-17 and TNF- $\alpha$ in human keratinocytes account for key inflammatory pathogenic circuits in psoriasis. J Invest Dermatol 131(3):677-687

19. Menter A et al (2008) Adalimumab therapy for moderate to severe psoriasis: A randomized, controlled phase III trial. J Am Acad Dermatol 58(1):106-115

20. Tyring $\mathrm{S}$ et al (2006) Etanercept and clinical outcomes, fatigue, and depression in psoriasis: double-blind placebo-controlled randomised phase III trial. Lancet 367:29-35

21. Papp KA et al (2005) A global phase III randomized controlled trial of etanercept in psoriasis: safety, efficacy, and effect of dose reduction. Br J Dermatol 152:1304-1312

22. Reich $\mathrm{K}$ et al (2005) Infliximab induction and maintenance therapy for moderate-to-severe psoriasis: a phase III, multicentre, doubleblind trial Kristian. Lancet 366:1367-1374

23. Reich K et al (2012) Successful treatment of moderate to severe plaque psoriasis with the PEGylated Fab $\varnothing$ certolizumab pegol: Results of a phase II randomized, placebo-controlled trial with a re-treatment extension. Br J Dermatol 167(1):180-190

24. Langley RG et al (2008) Efficacy and safety of ustekinumab, a human interleukin-12/23 monoclonal antibody, in patients with psoriasis: 52-week results from a randomised, double-blind, placebo-controlled trial (PHOENIX 2). Lancet 371:1675-1684

25. Blauvelt A et al (2017) Efficacy and safety of guselkumab, an antiinterleukin-23 monoclonal antibody, compared with adalimumab for the continuous treatment of patients with moderate to severe psoriasis: Results from the phase III, double-blinded, placebo- and active comparator. J Am Acad Dermatol 76(3):405-417

26. Reich K et al (2017) Tildrakizumab versus placebo or etanercept for chronic plaque psoriasis (reSURFACE 1 and reSURFACE 2): results from two randomised controlled, phase 3 trials. Lancet 390(10091):276-288

27. Papp K et al (2017) Risankizumab versus ustekinumab for moderate-to-severe plaque psoriasis. N Engl J Med 376(16): $1551-1560$

28. Reich K et al (2018) Ixekizumab Pharmacokinetics, Anti-Drug Antibodies, and Efficacy through 60 Weeks of Treatment of Moderate to Severe Plaque Psoriasis. J Invest Dermatol 138(10): 2168-2173

29. Langley RG et al (2014) Secukinumab in Plaque Psoriasis - Results of Two Phase 3 Trials. N Engl J Med 371(4):326-338

30. Papp KA et al (2016) A prospective phase III, randomized, doubleblind, placebo-controlled study of brodalumab in patients with moderate-to-severe plaque psoriasis. Br J Dermatol 175:273-286

31. Legat FJ, Hofer A, Quehenberger F, Kahofer P, Kerl H, Wolf P (2004) Reduction of treatment frequency and UVA dose does not substantially compromise the antipsoriatic effect of oral psoralenUVA. J Am Acad Dermatol 51(5):746-754

32. Yones SS, Palmer RA, Garibaldinos TT, Hawk JLM (2006) Randomized Double-blind Trial of the Treatment of Chronic Plaque Psoriasis. Arch Dermatol 142:836-842

33. Tanew A, Radakovic-Fijan S, Schemper M, Hönigsmann H (1999) Narrowband UV-B phototherapy vs photochemotherapy in the treatment of chronic plaque-type psoriasis: A paired comparison study. Arch Dermatol 135(5):519-524

34. Sivanesan SP et al (2009) Randomized, double-blind, placebocontrolled evaluation of the efficacy of oral psoralen plus ultraviolet 
A for the treatment of plaque-type psoriasis using the Psoriasis Area Severity Index score (improvement of $75 \%$ or greater) at 12 weeks. J Am Acad Dermatol 61(5):793-798

35. Inzinger $\mathrm{M}$ et al (2011) Efficacy of psoralen plus ultraviolet $\mathrm{A}$ therapy vs. biologics in moderate to severe chronic plaque psoriasis: Retrospective data analysis of a patient registry. Br J Dermatol 165(3):640-645

36. Mrowietz U et al (2015) Secukinumab retreatment-as-needed versus fixed-interval maintenance regimen for moderate to severe plaque psoriasis: A randomized, double-blind, noninferiority trial (SCULPTURE). J Am Acad Dermatol 73(1):27-36.e1

37. Blauvelt A et al (2017) Continuous dosing versus interrupted therapy with ixekizumab: an integrated analysis of two phase 3 trials in psoriasis. J Eur Acad Dermatol Venereol 31(6):1004-1013

38. Nakamura M et al (2017) Guselkumab for the Treatment of Psoriasis: A Review of Phase III Trials. Dermatol Ther (Heidelb) 7(3):281-292

39. Vallat VP et al (1994) PUVA bath therapy strongly suppresses immunological and epidermal activation in psoriasis: a possible cellular basis for remittive therapy. J Exp Med 180(1):283-296

40. Buhl T, Schön MP (2016) Peeking into immunoregulatory effects of phototherapy. Exp Dermatol 25(7):511-512

41. Morita A (2018) Current developments in phototherapy for psoriasis. J Dermatol 45(3):287-292

42. Vieyra-Garcia PA, Wolf P (2018) From Early Immunomodulatory Triggers to Immunosuppressive Outcome: Therapeutic Implications of the Complex Interplay Between the Wavebands of Sunlight and the Skin. Front Med 5(September):1-9

43. Wolf $P$ et al (2006) Platelet-activating factor is crucial in psoralen and ultraviolet A-induced immune suppression, inflammation, and apoptosis. Am J Pathol 169(3):795-805

44. Singh TP et al (2010) 8-Methoxypsoralen Plus Ultraviolet A Therapy Acts via Inhibition of the IL-23/Th17 Axis and Induction of Foxp3+ Regulatory T Cells Involving CTLA4 Signaling in a Psoriasis-Like Skin Disorder. J Immunol 184(12): $7257-7267$

45. Singh TP, Schön MP, Wallbrecht K, Gruber-Wackernagel A, Wang XJ, Wolf P (2013) Involvement of IL-9 in Th17-Associated Inflammation and Angiogenesis of Psoriasis. PLoS One 8(1):1-11

46. Johnson-Huang LM, Suárez-Farĩas M, Sullivan-Whalen M, Gilleaudeau P, Krueger JG, Lowes MA (2010) Effective narrowband UVB radiation therapy suppresses the IL-23/IL-17 Axis in normalized psoriasis plaques. J Invest Dermatol 130(11):2654-2663

47. Rácz E et al (2011) Effective treatment of psoriasis with narrowband UVB phototherapy is linked to suppression of the IFN and Th17 pathways. J Invest Dermatol 131(7):1547-1558

48. Wolf $\mathrm{P}$ et al (2000) Topical treatment with liposomes containing T4 endonuclease $\mathrm{V}$ protects human skin in vivo from ultravioletinduced upregulation of interleukin-10 and tumor necrosis factor$\alpha$. J Invest Dermatol 114(1):149-156

49. Singh TP, Schön MP, Wallbrecht K, Wolf P (2012) 8Methoxypsoralen plus UVA treatment increases the proportion of CLA+ CD25+ CD4+ T cells in lymph nodes of K5.hTGFb1 transgenic mice. Exp Dermatol 21:228-235

50. Schweintzger $\mathrm{N}$ et al (2015) Levels and function of regulatory $\mathrm{T}$ cells in patients with polymorphic light eruption: Relation to photohardening. Br J Dermatol 173(2):519-526

51. Wolf P et al (2014) Photohardening of polymorphic light eruption patients decreases baseline epidermal Langerhans cell density while increasing mast cell numbers in the papillary dermis. Exp Dermatol 23(6):428-430

52. Janssens AS, Pavel S, Out-Luiting JJ, Willemze R, De Gruijl FR (2005) Normalized ultraviolet (UV) induction of Langerhans cell depletion and neutrophil infiltrates after artificial UVB hardening of patients with polymorphic light eruption. Br J Dermatol 152(6): $1268-1274$
53. Weatherhead SC et al (2011) Keratinocyte apoptosis in epidermal remodeling and clearance of psoriasis induced by UV radiation. $\mathrm{J}$ Invest Dermatol 131(9): 1916-1926

54. Legat FJ et al (2007) Narrowband UV-B phototherapy, alefacept, and clearance of psoriasis. Arch Dermatol 143(8):1016-1022

55. Painsi $C$ et al (2015) Psoriasis Area and Severity Index 75 rate of classical inpatient dithranol therapy under daily life conditions. Br J Dermatol 173(3):815-817

56. Painsi $C$, Patscheider $M$, Inzinger $M$, Lange-Asschenfeldt $B$, Quehenberger F, Wolf P (2015) Patient perspectives on treating psoriasis with classic inpatient dithranol therapy: a retrospective patient survey. J Dtsch Dermatol Ges 13(11):1156-1163

57. Swinkels OQJ et al (2004) Effectiveness and side effects of UVBphototherapy, dithranol inpatient therapy and a care instruction programme of short contact dithranol in moderate to severe psoriasis. Eur J Dermatol 14(3):159-165

58. Griffiths CEM, van de Kerkhof P, Czarnecka-Operacz M (2017) Psoriasis and Atopic Dermatitis. Dermatol Ther 7(s1. Springer Healthcare):31-41

59. Tsoi LC et al (2012) Identification of 15 new psoriasis susceptibility loci highlights the role of innate immunity. Nat Genet 44(12):1341-1348

60. Zaba LC et al (2007) Amelioration of epidermal hyperplasia by TNF inhibition is associated with reduced Th17 responses. J Exp Med 204(13):3183-3194

61. Zaba LC et al (2009) Effective treatment of psoriasis with etanercept is linked to suppression of IL-17 signaling, not immediate response TNF genes. J Allergy Clin Immunol 124(5):102210.e1-395

62. Suárez-Farinas M, Fuentes-Duculan J, Lowes MA, Krueger JG (2011) Resolved psoriasis lesions retain expression of a subset of disease-related genes. J Invest Dermatol 131(2):391-400

63. Johnston A, Guzman AM, Swindell WR, Wang F, Kang S, Gudjonsson JE (2014) Early tissue responses in psoriasis to the anti-TNF- $\alpha$ biologic etanercept suggest reduced IL-17R expression and signalling. Br J Dermatol 171(1):97-107

64. Chow M, Lai K, Ahn R, Gupta R, Arron S (2016) Effect of adalimumab on gene expression profiles of psoriatic skin and blood. J Drugs Dermatol 15(8):988-994

65. Langkilde A et al (2016) Pathway analysis of skin from psoriasis patients after adalimumab treatment reveals new early events in the antiinflammatory mechanism of anti-TNF- $\alpha$. PLoS One 11(12):1-16

66. Bosè $\mathrm{F}$ et al (2013) Inhibition of CCR7/CCL19 axis in lesional skin is a critical event for clinical remission induced by TNF blockade in patients with psoriasis. Am J Pathol 183(2):413-421

67. Krueger JG et al (2012) IL-17A is essential for cell activation and inflammatory gene circuits in subjects with psoriasis. J Allergy Clin Immunol 130(1):145-154

68. Reich K et al (2015) Evidence that a neutrophil-keratinocyte crosstalk is an early target of IL-17A inhibition in psoriasis. Exp Dermatol 24(7):529-535

69. Kolbinger $F$ et al (2017) $\beta$-Defensin 2 is a responsive biomarker of IL-17A-driven skin pathology in patients with psoriasis. J Allergy Clin Immunol 139(3):923-932.e8

70. Russell CB et al (2014) Gene Expression Profiles Normalized in Psoriatic Skin by Treatment with Brodalumab, a Human Anti-IL-17 Receptor Monoclonal Antibody. J Immunol 192(8):3828-3836

71. Teng MWL et al (2015) IL-12 and IL-23 cytokines: From discovery to targeted therapies for immune-mediated inflammatory diseases. Nat Med 21(7):719-729

72. Brodmerkel C et al (2019) Modulation of inflammatory gene transcripts in psoriasis vulgaris: Differences between ustekinumab and etanercept. J Allergy Clin Immunol 143(5):1965-1969

73. Visvanathan S et al (2019) Psoriatic skin molecular and histopathologic profiles after treatment with risankizumab versus ustekinumab. J Allergy Clin Immunol 143(6):2158-2169 
74. Sofen H et al (2014) Guselkumab (an IL-23-specific mAb) demonstrates clinical and molecular response in patients with moderateto-severe psoriasis. J Allergy Clin Immunol 133(4):1032-1040

75. Kulig P et al (2016) IL-12 protects from psoriasiform skin inflammation. Nat Commun 7(1):13466

76. Cheuk S et al (2014) Epidermal Th22 and Tc17 Cells Form a Localized Disease Memory in Clinically Healed Psoriasis. J Immunol 192(7):3111-20

77. Cheuk S et al (2017) CD49a Expression Defines Tissue-Resident CD8 + T Cells Poised for Cytotoxic Function in Human Skin. Immunity 46:287-300

78. Matos TR et al (2017) Clinically resolved psoriatic lesions contain psoriasis-specific IL-17-producing $\alpha \beta$ T cell clones. J Clin Invest 127(11):4031-4041

79. Gallais Sérézal I et al (2018) Resident T Cells in Resolved Psoriasis Steer Tissue Responses that Stratify Clinical Outcome. J Invest Dermatol 138(8):1754-1763

80. Gallais Sérézal I et al (2019) A skewed pool of resident T cells triggers psoriasis-associated tissue responses in never-lesional skin from patients with psoriasis. J Allergy Clin Immunol 143(4):1444-1454

81. Eidsmo L, Martini E (2018) Human Langerhans cells with proinflammatory features relocate within psoriasis lesions. Front Immunol 9(300):1-8

82. Singh TP et al (2016) Monocyte-derived inflammatory Langerhans cells and dermal dendritic cells mediate psoriasis-like inflammation. Nat Commun 7(May):13581

83. Martini E et al (2017) Dynamic Changes in Resident and Infiltrating Epidermal Dendritic Cells in Active and Resolved Psoriasis. J Invest Dermatol 137(4):865-873

84. Choi JE, Di Nardo A (2018) Skin Neurogenic Inflammation. Semin Immunopathol 40(3):6072-6078

85. Wolf P, Weger W, Patra VK, Gruber-Wackernagel A, Byrne SN (2016) Desired response to phototherapy vs photoaggravation in psoriasis: what makes the difference? Exp Dermatol 25(12):937-944
86. Patra VK, Mayer G, Gruber-Wackernagel A, Horn M, Lembo S, Wolf P (2018) Unique profile of antimicrobial peptide expression in polymorphic light eruption lesions compared to healthy skin, atopic dermatitis, and psoriasis. Photodermatol Photoimmunol Photomed 34(2):137-144

87. Sugiura K et al (2013) The majority of generalized pustular psoriasis without psoriasis vulgaris Is caused by deficiency of interleukin-36 receptor antagonist. J Invest Dermatol 133(11):2514-2521

88. Johnston A et al (2017) IL-1 and IL-36 are dominant cytokines in generalized pustular psoriasis. J Allergy Clin Immunol 140(1):109-120

89. Buhl AL, Wenzel J (2019) Interleukin-36 in infectious and inflammatory skin diseases. Front Immunol 10(May):1-11

90. Li N et al (2014) Alarmin Function of Cathelicidin Antimicrobial Peptide LL37 through IL-36 $\gamma$ Induction in Human Epidermal Keratinocytes. J Immunol 193(10):5140-5148

91. Furue $\mathrm{K}$ et al (2018) Highlighting interleukin-36 signalling in plaque psoriasis and pustular psoriasis. Acta Derm Venereol 98(1):5-13

92. Ngo VL et al (2018) A cytokine network involving IL-36 $\gamma$, IL-23, and IL-22 promotes antimicrobial defense and recovery from intestinal barrier damage. Proc Natl Acad Sci U S A 115(22):E5076-E5085

93. Madonna S, Girolomoni G, Dinarello CA, Albanesi C (2019) The Significance of IL-36 Hyperactivation and IL-36R Targeting in Psoriasis. Int J Mol Sci 20(3318):1-14

94. Bachelez H et al (2019) Inhibition of the Interleukin-36 Pathway for the Treatment of Generalized Pustular Psoriasis. N Engl J Med 380(10):981-983

95. Mahil SK et al (2017) An analysis of IL-36 signature genes and individuals with IL1RL2 knockout mutations validates IL-36 as a psoriasis therapeutic target. Sci Transl Med 9 (411):eaan2514

Publisher's note Springer Nature remains neutral with regard to jurisdictional claims in published maps and institutional affiliations. 